\title{
Targeted Mutagenesis in the Study of the Tight Adherence (tad) Locus of Aggregatibacter actinomycetemcomitans
}

\author{
David H. Figurski1, Daniel H. Fine ${ }^{2}$, Brenda A. Perez-Cheeks ${ }^{1}$, \\ Valerie W. Grosso' ${ }^{1}$ Karin E. Kram¹, Jianyuan Hua ${ }^{1}$, \\ $\mathrm{Ke} \mathrm{Xu}^{1}$ and Jamila Hedhli ${ }^{1}$ \\ ${ }^{1}$ Department of Microbiology \& Immunology, College of Physicians \& Surgeons, \\ Columbia University, New York, NY, USA \\ ${ }^{2}$ Department of Oral Biology, The University of Medicine \& Dentistry of New Jersey, \\ Newark, NJ, USA
}

\section{Introduction}

Aggregatibacter actinomycetemcomitans is a Gram-negative, capnophilic ( $\mathrm{CO}_{2}$ loving), coccobacillus found only in humans and Old World primates (for reviews, see Henderson et al., 2003, 2010). This bacterium is primarily known as the etiologic agent of Localized Aggressive Periodontitis (LAP), which is predominantly an infection of adolescents (Slots \& Ting, 1999; Zambon, 1985). A. actinomycetemcomitans also causes extraoral infections, including infective endocarditis, septicemia, and abscesses (Fine et al., 2006; Fives-Taylor et al., 1999; Rahamat-Langendoen et al., 2011; van Winkelhoff \& Slots 1999). A. actinomycetemcomitans is a member of the HACEK group of Gram-negative bacteria, all of which can cause infective endocarditis (Paturel et al., 2004). Most HACEK bacteria-caused cases of infective endocarditis result from A. actinomycetemcomitans. Poor oral health is a risk factor for developing severe extraoral infections by A. actinomycetemcomitans (Paturel et al., 2004; van Winkelhoff \& Slots 1999). One study showed that $16 \%$ of patients with infective endocarditis from $A$. actinomycetemcomitans had dental procedures immediately prior to the onset of disease. A staggering $42 \%$ of A. actinomycetemcomitans-caused infective endocarditis patients had generalized dental disease - an indication of poor overall oral health.

LAP is a severe form of periodontitis (Genco et al., 1986; Slots \& Ting, 1999). For reasons so far unknown, the disease is localized to the premolar and incisor teeth. Infection leads to inflammation and rapid destruction of the periodontal ligament and the alveolar bone and culminates in loss of teeth. The prevalence of LAP has been estimated to be $0.5-1 \%$ (Henderson et al., 2002; Löe \& Brown, 1991; Rylev \& Kilian, 2008). However, prevalence varies considerably with different ethnic groups. For example, in the African-American population, the prevalence is 10-15 times higher than the average. There is evidence that race and socioeconomic status play key roles in determining prevalence.

The molecular mechanisms behind the pathogenesis of A. actinomycetemcomitans are not understood. The organism elaborates a number of factors that have been implicated in 
virulence (Fine et al., 2006; Fives-Taylor et al., 1999). Several adhesins have been identified. The best studied adhesins are (1) Aae, an autotransporter that binds to buccal epithelial cells (Fine et al., 2005, 2010; Rose et al., 2003); (2) ApiA, another autotransporter that binds to buccal epithelial cells, but with lower affinity than does Aae (Komatsuzawa et al., 2002; Yue et al., 2007); and (3) EmaA, which binds collagen (Jiang et al., 2012; Mintz, 2004). A leukotoxin affects leukocytes by inducing one of several pathways (Tsai et al., 1979; Kachlany, 2010). The pathway that is activated depends on the cell type. The toxic effects include apoptosis, degranulation, and a novel lysosome-mediated cell-death pathway (DiFranco et al., 2012; Fong et al., 2006; Kelk et al., 2003, 2011; Lally et al., 1999). Another factor is the cytolethal distending toxin, which causes death by cell-cycle arrest (De Rycke \& Oswald, 2001; Fine et al., 2006; Pickett \& Whitehouse, 1999).

\subsection{Non-specific adherence and virulence}

A particularly striking property of a fresh clinical isolate of A. actinomycetemcomitans is its ability to form an extremely tenacious biofilm on inert surfaces, such as glass, plastic, and hydroxyapatite (Fig. 1) (Fine et al., 1999a; Kachlany et al., 2000, 2001a). Freshly isolated clinical strains of A. actinomycetemcomitans form small, rough colonies on agar plates (Fig. 2A). In broth, the clinical isolates tightly adhere to the surfaces of culture vessels; and aggregates may be visible at the bottom of a tube. The cells express long, bundled, protein fibrils, termed "Flp fibers," which are required to form the tenacious biofilm. (A Flp fiber is composed of several Flp pili.) Propagation of adherent wild-type strains generally leads to spontaneous variants (see Section 4 below) that produce smooth colonies (Fig. 2B), have cells that do not autoaggregate, and cannot produce tenacious biofilms because the cells do not express Flp pili (Fig. 1, right) (Fine et al., 1999b; Kachlany et al., 2001a).

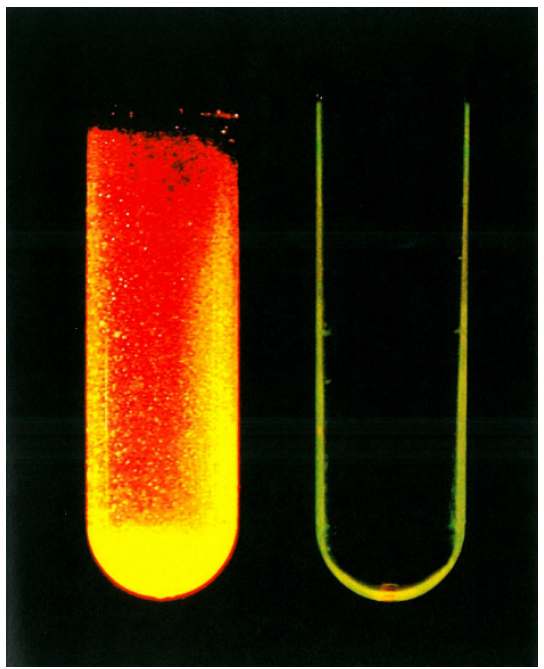

Fig. 1. Tenacious adherence of A. actinomycetemcomitans.

Cultures of a clinical isolate (left) and a spontaneous smooth-colony variant (right) were grown in broth. After the culture was mixed and the broth was poured out, the remaining adherent cells were stained with ethidium bromide and illuminated with UV light. 
Studies in our LAP-infection model demonstrated that a wild-type strain is able to colonize and persist in the mouths of rats (Fine et al., 2001). In contrast, an isogenic smooth-colonyforming variant failed to persist. Despite this unequivocal evidence for the importance of tenacious adherence in the colonization of the oral cavity by A. actinomycetemcomitans, the genetic and molecular bases underlying this remarkable property were unknown.

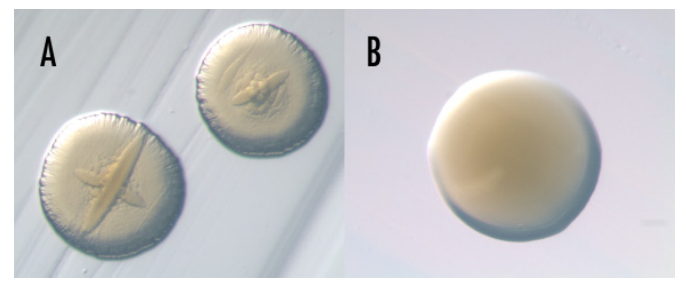

Fig. 2. Colony morphologies of A. actinomycetemcomitans.

(A) "Rough" colony morphology of a clinical isolate. (B) "Smooth" colony morphology of a spontaneous variant.

\subsection{The tad locus}

The study of A. actinomycetemcomitans had been hampered by a paucity of molecular tools for genetic manipulation, especially tools for clinical isolates. We adapted the transposon Tn903 to provide inducible random mutagenesis of the chromosome of $A$. actinomycetemcomitans (Thomson et al., 1999). A clinical isolate of A. actinomycetemcomitans from a 13-year-old African-American female was mutagenized using our synthetic Tn903 transposon (IS903фkan). The random-mutant bank was screened for mutant strains that formed smooth colonies. Identifying and sequencing the transposon insertion sites of smooth mutants led to the identification of a 14-gene segment, designated the tad (tight adherence) locus (Fig. 3A) (Kachlany et al., 2000; Planet et al., 2003; Tomich et al, 2007). The tad locus encodes a novel macromolecular transport system that is used in the biogenesis of Flp pili (Fig. 4). One hypothetical gene of the tad locus, flp-2, is not needed for Flp pili

A

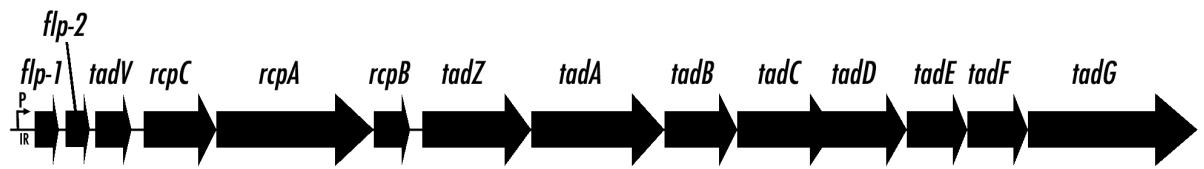

B

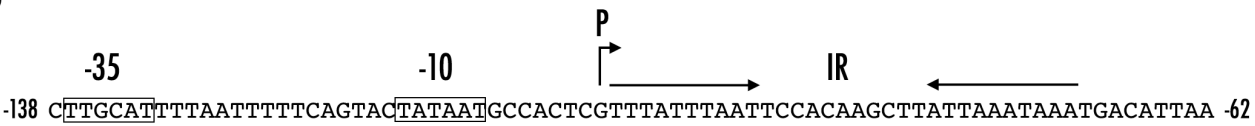

Fig. 3. The tad locus of A. actinomycetemcomitans.

(A) The size of the locus is approximately $12 \mathrm{~kb}$. Filled arrows designate the genes. Their relative sizes are approximately correct. " $\mathrm{P}$ " and the bent arrow indicate the transcriptional start site (Haase et al., 2003); "IR," inverted repeat. (B) The promoter region magnified. 
biosynthesis in A. actinomycetemcomitans (Perez et al., 2006). We determined whether the gene products are cytoplasmic, in the inner membrane, or in the outer membrane (Clock et al., 2008). Our studies have also revealed the functions or features of the products of several tad locus genes (Tomich et al., 2007). flp-1 encodes the Flp1 prepilin (Inoue et al., 1998; Kachlany et al., 2001b); tadV encodes a protease that processes the Flp1 prepilin to the mature Flp1 pilin that is needed for assembly into Flp pili (Tomich et al., 2006); the product of $r c p A$ (Haase et al., 1999) has the properties of an outer membrane pore (secretin) (Clock et al., 2008); the product of $r c p B$ (Haase et al., 1999) is an outer-membrane protein that may gate the pore (Clock et al., 2008; Perez et al., 2006); tadZ encodes a protein that may localize the Tad secretion machine to a pole (Perez-Cheeks et al., 2012; Xu et al., 2012; see Section 3 below); the tadA product is an ATPase (Bhattacharjee et al., 2001); and the tadE and tadF products are "pseudopilins," whose functions are not known; but the pseudopilins are processed by TadV in the same way that the prepilin is (Tomich et al., 2006).

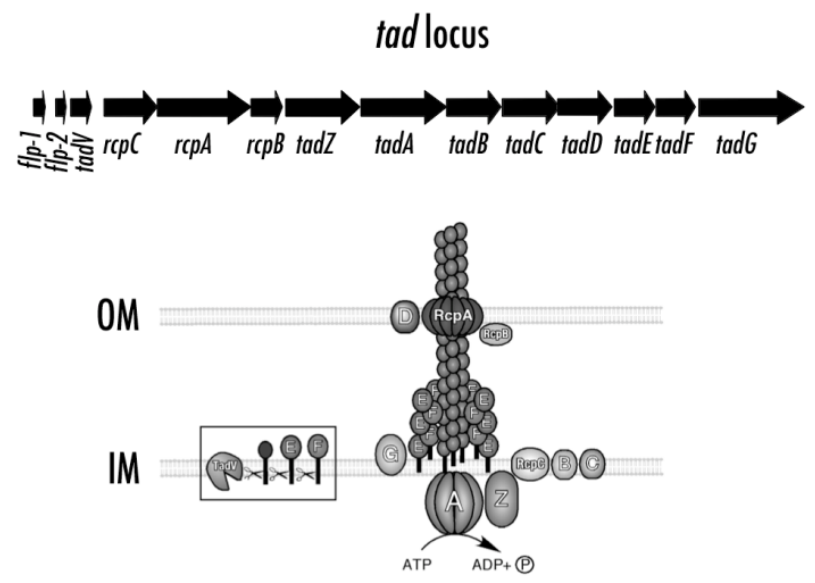

Fig. 4. Hypothetical structure of the Tad secretion apparatus.

Shown is a cartoon of the Flp pilus and its secretion machine formed (at least in part) by a complex of the tad gene products. The lack of detail reflects the paucity of data for the gene products. "IM" means "inner membrane"; "OM," "outer membrane."

\subsection{The tad locus in A. actinomycetemcomitans is a virulence factor}

Using our rat model of LAP, we demonstrated that a functional tad locus is needed for the ability of A. actinomycetemcomitans to colonize and persist in the oral cavity and to cause tissue and bone destruction (Schreiner et al., 2003). Unlike the wild-type strain, isogenic tad locus mutant strains, defective in either flp-1 (encoding the pilin) or tadA (encoding an ATPase), did not persist in the mouths of the rats; nor did they cause bone loss. In addition to our evidence in A. actinomycetemcomitans, studies in other bacterial species have demonstrated the involvement of tad homologs in virulence: Haemophilus ducreyi (Spinola et al., 2003), the etiologic agent of chancroid, a sexually transmitted disease, and Pasteurella multocida (Fuller et al., 2000), which causes fowl cholera. The tad genes of Pseudomonas aeruginosa are needed for biofilm formation and for adherence to epithelial cells (de Bentzmann et al., 2006). 


\section{4 tad loci are widespread in prokaryotes}

Searches of completed and ongoing microbial sequencing projects have revealed that closely related tad gene clusters are present in the genomes of a wide variety of Gram-negative and Gram-positive bacteria (Kachlany et al., 2001a; Tomich et al, 2007; P. Planet \& D. Figurski unpublished results, P. Planet, unpublished results), some of which represent significant threats to human health. Among the pathogens are Yersinia pestis, the agent of bubonic plague; Mycobacterium tuberculosis, the agent of tuberculosis; Bordetella pertussis, the agent of whooping cough; Burkhoderia cepacia, a frequent colonizer of the lungs of patients with cystic fibrosis; and P. aeruginosa, an opportunistic human pathogen. About $40 \%$ of over 3400 bacterial genomes sequenced to date have tad loci. In addition, potentially all Archaea may harbor homologs of tad genes (P. Planet, personal communication). Given our evidence that the tad locus is foreign to the chromosome of A. actinomycetemcomitans and that tad loci are widely distributed in prokaryotes, we have also referred to the tad locus as the "Widespread Colonization Island" (WCI) (Planet et al., 2003).

\section{The FIp pilus}

The Flp pili of A. actinomycetemcomitans (Fig. 5) are proteinaceous fibers that are attached to the exterior of the bacterial cell and are necessary for tenacious adherence (Kachlany et al., 2000, 2001b). Flp pili are polymers of the mature Flp1 pilin protein, and they are assembled and secreted by a complex of proteins encoded by the tad locus. Flp pili are abundant, extremely adhesive, and bundled. The $f l p-1$ and $f l p-2$ genes of A. actinomycetemcomitans and their predicted products and the flp genes from other organisms and their predicted products form a distinct, monophyletic group with homology to other pilin genes, particularly to those of subclass b (Kachlany et al., 2001b). We (M. Tomich and D. Figurski, unpublished results) and others (Inoue et al., 2000) have shown that the Flp1 pilin is a glycoprotein, but the structure and function of the modification is unknown.

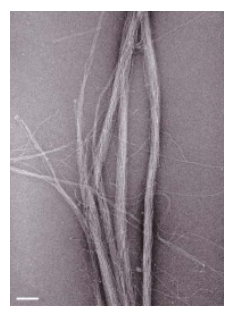

Fig. 5. Flp pili.

Transmission electron micrograph of purified Flp pili from A. actinomycetemcomitans.

After their translocation to the inner membrane, the prepilins are cleaved (processed) to maturity by cognate prepilin peptidases (Giltner et al., 2012). We have shown that the 75amino acid Flp1 prepilin of A. actinomycetemcomitans is cleaved by TadV protein at the sequence $\mathrm{G}^{\wedge} \mathrm{XXXXEY}$ (Tomich et al., 2006). The mature Flp1 pilin is only 49 amino acids in length, which is much smaller than other known type IV pilins (Giltner et al., 2012; Kachlany et al., 2001b). Because of its small size, we believe Flp1 pilin is an attractive subject for genetic and structural analysis. In addition to learning about the molecular details of Flp1, we also wish to understand the basis of the three most obvious phenotypes of Flp pili: 
tenacious adherence to surfaces, binding to A. actinomycetemcomitans cells, and binding to each other (bundling).

\subsection{Alanine-scanning mutagenesis of the coding region for the mature Flp1 pilin}

To begin to study the properties of Flp1, we constructed and characterized a series of Flp1 pilin mutants, each with an alanine substitution for a specific non-alanine residue of the mature Flp1 pilin. The codon for each non-alanine residue was changed to a codon for alanine. (The mutant genes were constructed with the fewest possible nucleotide changes.) In this way, translation of the mutant gene would give a mutant Flp1 prepilin, which, after being processed, gave rise to a mutant mature Flp1 pilin. (Alanine was chosen because it is the smallest amino acid that is relatively neutral and can maintain an $\alpha$-helix in a polypeptide.) We changed the non-alanine residues in the mature Flp1 pilin by overlap extension PCR (polymerase chain reaction) (Ho et al., 1989). In this method, the flp-1 gene was divided into two segments, each of which could be amplified by PCR with a pair of primers. For each segment, one primer annealed just beyond an end of the gene; the other primer was directed to the internal region of the gene where the mutation was to be introduced. The internal primers for the two segments carried the appropriately changed nucleotides. As a result, the two amplified segments overlapped slightly. When the two segments were added together, denatured, and reannealed, a single strand of one segment could anneal in the overlap region with the opposite strand of the other segment. One half of the hybrid molecules would have free $5^{\prime}$ ends facing the single-stranded portion. These are dead-ends. The other half would have free $3^{\prime}$ ends, which could prime DNA synthesis to give full-length duplex molecules with the mutation in both strands. By using the endspecific primers, the full-length, mutated $f l p-1$ gene could be amplified and cloned. The mutation was then confirmed in the clone by nucleotide sequencing.

\subsection{Characterization of mutant Flp1 pilins}

The mutant genes were inserted into a plasmid vector downstream of the IPTG (isopropyl $\beta$ D-thiogalactopyranoside)-inducible tac promoter. (The tac promoter is a strong promoter that is a hybrid of the trp and lac promoters. Like the lac promoter, the tac promoter is inhibited by the Escherichia coli LacI repressor protein, whose gene was already added to the plasmid cloning vector. LacI is inactivated by IPTG.) We wanted to know (1) if the mutant pilin had wild-type abundance, (2) if the mutant flp-1 gene could complement a flp-1 chromosomal mutant gene, (3) if the mutant pilin allowed Flp pili to be made, and (4) if any Flp pili assembled with the mutant pilin promoted adherence. Mutant Flp1 pilin abundance was indicated by immunological detection of pilin in protein extracts that were separated into bands by electrophoresis in a sodium dodecyl sulfate polyacrylamide gel (Western blot). Genetic complementation was detected by the conversion of the smooth-colony morphology of a flp-1- mutant strain to a rough-colony morphology reminiscent of the wildtype strain. The presence of Flp pili was shown by electron microscopy of $f l p-1$ - mutant cells carrying the mutant pilin gene. To quantify adherence, a slightly modified crystal violet assay of O'Toole and Kolter (1998) was used. Basically, wells of microtiter dishes were inoculated with the strains to be tested. Nonadherent cells were removed by washes. Adherent cells were then stained with crystal violet. After more washes to remove free crystal violet and any remaining nonadherent cells, the crystal violet in the adherent cells 
was then eluted with DMSO (dimethyl sulfoxide). The eluted crystal violet gave a color to the solution that was quantified with a spectrophotometer. Increasing crystal violet in the eluate is indicative of increasing adherence.

Each of our Flp1 mutants had one non-alanine residue changed to alanine. (See Table 1 for the single-letter and three-letter codes for the amino acids. Table 2 shows the residue change in the Flp1 pilin for each mutant and the phenotype.) In the mutants, every non-alanine residue of the mature Flp1 pilin was changed to alanine. The Flp1 prepilin has 75 residues; but, after cleavage, the mature Flp1 pilin has 49 residues. Nine residues are already alanine. The other 40 residues of the mature pilin were changed to alanine. The small size of mature Flp1 pilin made it reasonable to create this series of mutant pilins.

\begin{tabular}{|l|c|c|}
\hline Amino acid & Single-letter code & Three-letter code \\
\hline alanine & A & ala \\
\hline arginine & R & arg \\
\hline asparagine & N & asn \\
\hline aspartic acid & D & asp \\
\hline cysteine & C & glu \\
\hline glutamic acid & E & gln \\
\hline glutamine & Q & gly \\
\hline glycine & G & his \\
\hline histidine & H & ile \\
\hline isoleucine & I & leu \\
\hline leucine & L & lys \\
\hline lysine & K & met \\
\hline methionine & M & phe \\
\hline phenylalanine & F & pro \\
\hline proline & P & ser \\
\hline serine & S & thr \\
\hline threonine & T & try \\
\hline tryptophan & W & tyr \\
\hline tyrosine & Y & val \\
\hline valine & V & \\
\hline
\end{tabular}

Table 1. Single-letter and three-letter codes for the amino acids.

\begin{tabular}{ccccccc} 
Mutant Residue & $\begin{array}{c}\text { Colony } \\
\text { Morph. }^{\mathrm{a}}\end{array}$ & Adher. $^{\mathrm{b}}$ & Auto-aggreg. $^{\mathrm{c}}$ & $\begin{array}{c}\text { Protein } \\
\text { exp. }^{\text {d }}\end{array}$ & Piliation $^{\mathrm{e}}$ & Class $^{\mathrm{f}}$ \\
\hline V27A & S & - & - & $+/-$ & + & IV \\
T28A & S & - & - & + & - & III \\
I30A & S & - & - & + & + & IV \\
E31A & S & - & - & + & - & III \\
Y32A & S & - & - & $+/-$ & - & III \\
G33A & R & + & + & ND & ND & I
\end{tabular}




\begin{tabular}{|c|c|c|c|c|c|c|}
\hline Mutant Residue & $\begin{array}{l}\text { Colony } \\
\text { Morph. }^{a}\end{array}$ & Adher. ${ }^{b}$ & Auto-aggreg.c & $\begin{array}{c}\text { Protein } \\
\text { exp. }^{\mathrm{d}}\end{array}$ & Piliation ${ }^{\mathrm{e}}$ & Class $^{f}$ \\
\hline L34A & S & - & - & $+/-$ & + & IV \\
\hline $\mathrm{I} 35 \mathrm{~A}$ & $S$ & - & - & - & - & II \\
\hline I37A & $S$ & - & - & + & - & III \\
\hline V39A & $\mathrm{R}$ & + & + & ND & ND & $\mathrm{I}$ \\
\hline V41A & $\mathrm{R}$ & + & + & ND & ND & I \\
\hline L42A & $\mathrm{R}$ & + & + & ND & ND & $\mathrm{I}$ \\
\hline $\mathrm{I} 43 \mathrm{~A}$ & $S$ & - & - & + & + & IV \\
\hline $\mathrm{V} 44 \mathrm{~A}$ & $\mathrm{R}$ & + & + & ND & ND & I \\
\hline V46A & $S$ & - & - & + & + & IV \\
\hline F47A & $S$ & - & - & + & - & III \\
\hline Y48A & $\mathrm{R}$ & + & + & ND & ND & I \\
\hline S49A & $\mathrm{R}$ & + & + & ND & ND & I \\
\hline N50A & $S$ & - & - & + & - & III \\
\hline N51A & $\mathrm{R}$ & + & + & ND & ND & $\mathrm{I}$ \\
\hline G52A & $S$ & - & - & + & + & IV \\
\hline F53A & $\mathrm{R}$ & + & + & ND & ND & I \\
\hline $\mathrm{I} 54 \mathrm{~A}$ & $S$ & - & - & - & - & II \\
\hline N56A & $\mathrm{R}$ & + & + & ND & ND & $\mathrm{I}$ \\
\hline L57A & $S$ & - & - & + & + & IV \\
\hline Q58A & $\mathrm{R}$ & + & + & ND & ND & I \\
\hline S59A & $\mathrm{R}$ & + & + & ND & ND & I \\
\hline K60A & $\mathrm{R}$ & + & + & ND & ND & I \\
\hline F61A & $S$ & - & - & + & - & III \\
\hline N62A & $\mathrm{R}$ & + & + & ND & ND & I \\
\hline S63A & $\mathrm{R}$ & + & + & ND & ND & I \\
\hline L64A & $S$ & - & - & + & - & III \\
\hline S66A & $\mathrm{R}$ & + & + & ND & ND & I \\
\hline T67A & $\mathrm{R}$ & + & + & ND & ND & I \\
\hline V68A & $\mathrm{R}$ & + & + & ND & ND & I \\
\hline S70A & $\mathrm{R}$ & + & + & ND & ND & I \\
\hline N72A & $\mathrm{R}$ & + & + & ND & ND & I \\
\hline V73A & $S$ & - & - & $+/-$ & - & III \\
\hline $\mathrm{T} 74 \mathrm{~A}$ & $\mathrm{R}$ & + & + & ND & ND & I \\
\hline K75A & $S$ & - & - & $+/-$ & + & IV \\
\hline
\end{tabular}

Table 2. Flp1 mutants and phenotypes.

a $\mathrm{R}$, rough colony morphology; $\mathrm{S}$, smooth colony morphology; $\mathrm{b}+, \geq 60 \%$ wild-type adherence; -, $<60 \%$ wild-type adherence; $c+$, autoaggregation; -, no autoaggregation; $d+$, wild-type Flp1 level; +/-, intermediate Flp1 level; -, no Flp1 observed; e +, pili observed; -, no pili observed; $\mathrm{ND}=$ not determined; $\mathrm{f}$ I-IV , phenotypic class 
The mutant pilins were assayed for the phenotypes described above. We divided the mutant pilins into four phenotypic classes (Tables 2 and 3, Fig. 6). Class I Flp1 pilin mutants (21 in number) were indistinguishable from the wild-type pilin in our assays. In other in vitro assays or in animal experiments, some of these Class I mutants might show phenotypes that differ from those of the wild-type pilin. Six of the seven residues in the hydrophobic-region Class I mutants were originally glycine (1), valine (4), and leucine (1), all of which are similar to the alanine replacement. The seventh mutant in the hydrophobic region had alanine in place of tyrosine, a larger hydrophobic residue. There were two Class II mutants defined as showing no or very little pilin. Class III (9) and Class IV (8) mutants all showed abundant pilin, approximately equal to the abundance of the wild type pilin. However, electron micrographs showed that Class III pilins do not form pili. In contrast, Class IV mutant pilins could be assembled into pili; but the pili were nonadherent. Consequently, Class IV mutant cells did not autoaggregate, nor could a Class IV mutant strain form the tenacious Tad biofilm. One of the Class IV mutants (K75A) is particularly interesting because it produces curved and non-bundled pili.

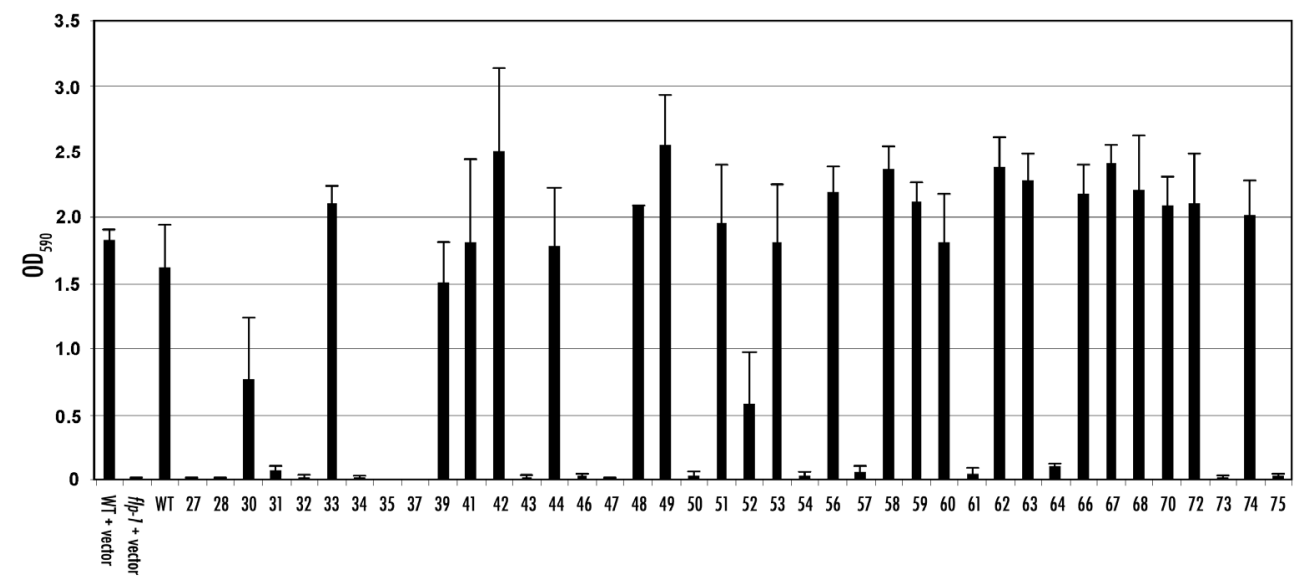

Fig. 6. Effect of mutant Flp1 pilins on adherence by A. actinomycetemcomitans.

Shown are the results of crystal violet assays for adherence. The first three results on the left are the controls: (1) positive controls, which are the wild-type A. actinomycetemcomitans strain (WT) with and without the empty plasmid used for cloning (vector) and (2) a negative control, which is a Flp1 pilin mutant strain $(f l p-1)$ with the empty vector. The mutant strain does not show tenacious adherence because it cannot make Flp pili. The numbers on the Xaxis indicate the residues that were changed to alanine. The $\mathrm{OD}_{590}$ values on the Y-axis indicate relative amounts of crystal violet eluted from adherent cells (see text). The error bars show the calculated ranges of values from three experiments.

Because there is no 3D structure yet for Flp1 pilin, we used structure-prediction software with the sequence. Like other type IV pilins, the mature Flp1 pilin was predicted to be divided into a largely hydrophobic N-terminal domain and a distinct C-terminal domain. In Flp1, the C-terminal domain was predicted to contain an 11-residue amphipathic $\alpha$-helix and a 12-residue, mostly-polar, C-terminal tail. It is thought that pilin subunits interact for polymerization in the hydrophobic region (Giltner et al., 2012). We therefore expected the 
"assembly" mutants (Class III) to be caused by alanine substitutions of residues predominantly in the hydrophobic $\mathrm{N}$-terminal region. Conversely, the C-terminal region of a pilin is thought to interact with the environment (Giltner et al., 2012), so we expected Class IV mutants to occur mostly from alterations of C-terminal residues. However, we were somewhat surprised by the number of Class III mutants with a substitution in a C-terminal residue, and by the number of Class IV mutants with a change in an $\mathrm{N}$-terminal residue.

\begin{tabular}{ccccc}
$\begin{array}{c}\text { Mutant } \\
\text { Class }\end{array}$ & Adherence & $\begin{array}{c}\text { Protein } \\
\text { Expression }\end{array}$ & Piliation & $\begin{array}{c}\text { Pilus } \\
\text { Morphology }\end{array}$ \\
\hline I & + & ND & ND & ND \\
II & - & - & ND & ND \\
III & - & + & - & NA \\
IV & - & + & + & $\begin{array}{c}\text { Normal and } \\
\text { altered }\end{array}$ \\
\hline
\end{tabular}

NA, "not applicable"; ND, "not determined."

Table 3. Summary of Flp1 mutant classes.

A previous study indicated that seven serine and asparagine residues in the C-terminal region are modified (Inoue et al., 2000). We found that only one substitution of those seven residues, a Class III mutant (N50A), gave a mutant phenotype. We do not know if the defect is due to the loss of modification at this residue or to the change in that amino acid.

Alanine-scanning mutagenesis has been an important step in beginning to understand the Flp1 pilin. We now have a collection of mutant pilins that can be studied for information on pilin stability, pili assembly, pili bundling, and pili adherence. These mutants will guide experiments in which substitutions can be made with amino acids that are of different sizes, have similar properties, or have very different properties. Similar experiments can also be done at the alanine residues.

A 3D structure of the Flp1 pilin is needed. There are a few structures of type IV pilins, most of which were determined from crystals formed after removing the N-terminal hydrophobic domain (Giltner et al., 2012). The Flp1 structure will clearly be different from the few structures of other type IV pilins. For example, Flp1 is 2 to 3-fold smaller than other type IV pilins. Also the mature Flp1 pilin has no cysteine residues, which are thought to be needed to form a disulfide bond to make the $\mathrm{D}$ region structure that seems to be conserved in type IV pilins. The phenotypes of the Flp1 mutants have also underscored the differences of Flp1 pilin and the "typical" type IV pilins. A structure would help us to understand (1) what is truly "typical" in type IV pilins, (2) the importance of certain Flp1 residues, and (3) the molecular basis of the phenotypes of the mutants.

\section{TadZ}

The $\operatorname{tadZ}$ gene has no known homolog, and it is unique to tad loci. The presence of a tadZ homolog is taken to indicate that a series of genes is, is part of, or once was a tad locus. In the tad locus of A. actinomycetemcomitans, tadZ is essential for the biogenesis of Flp pili and, therefore, for the tenacious biofilm. Our recent fluorescence-microscopy study of a TadZ- 
EGFP (enhanced green fluorescent protein) fusion showed that TadZ protein localizes to the old cell pole (i.e., opposite the pole formed by cell division) (Fig. 7) (Perez-Cheeks et al., 2012). It localized in the absence of any other protein encoded by the tad locus. (The TadZEGFP fusion was formed by eliminating the translational stop codon of the tadZ gene and attaching it in-frame to the coding sequence for EGFP minus its ribosome-binding-site and translational start codon.) In contrast, a TadA-EGFP fusion also localized to a pole, but only when TadZ protein was present. We proposed that TadZ is responsible for localizing the entire Tad secretory apparatus to a pole (Perez-Cheeks et al., 2012).

We did a large phylogenetic analysis and showed that the tadZ genes belong in the parA/minD superfamily of genes (Perez-Cheeks et al., 2012). The prototypical bacteriophage P1 ParA protein (and each of the various ParA-like proteins) is needed for the proper segregation of DNA at cell division. The E. coli MinD protein, which is thought to be the prototype for the other MinD-like proteins encoded by the family, has been studied extensively. It is needed to localize the septum properly at cell division. Other gene families in the parA/minD superfamily are the nifH family (named for nitrogen fixation), the fleN family (named for flagellar synthesis), and the $b c s Q$ family (named for cellulose biosynthesis).
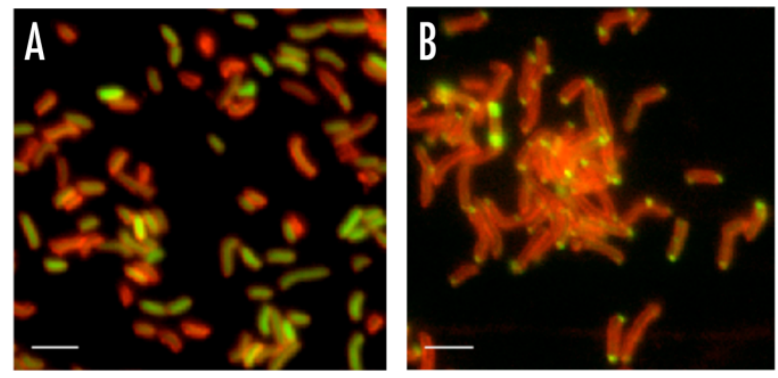

Fig. 7. Polar foci of the TadZ-EGFP fusion protein.

Shown are micrographs of tadZ mutant cells of A. actinomycetemcomitans slightly elongated by treatment with a sub-inhibitory concentration of the antibiotic piperacillin, stained with the membrane-specific fluorescent dye TMA-DPH [1-(4-trimethylammoniumphenyl)-6phenyl-1,3,5-hexatriene p-toluenesulfonate] (fluoresces red), and containing a plasmid expressing (A) green fluorescent protein as a control or (B) a TadZ-EGFP fusion. The cells were illuminated with UV light. Polar foci are seen only in the cells expressing the TadZEGFP fusion protein (Perez-Cheeks et al., 2012).

\subsection{The atypical Walker-like $A$ box of TadZ proteins}

Each of the protein products of the $\operatorname{par} A / \min D$ superfamily of genes has a Walker-like A box [KGGXX(S/T)] (Fig. 8), which forms a structure involved in binding and hydrolyzing ATP. The Walker-like A box is a variation of the Walker A box [GXXGXGK(S/T)], which also allows a protein to bind and hydrolyze ATP. However, the ATPases of Walker A box proteins are considerably stronger than the ATPases of the Walker-like A box proteins. Each product of the tadZ gene family also has a Walker-like A box, but the TadZ Walker-like A box is unique. The second lysine (K6 in the numbering system of Fig. 8) is missing. We call 
the unique Walker-like A motif in TadZ proteins the "atypical Walker-like A box" (PerezCheeks et al., 2012).

$\begin{array}{llllllll} & 1 & 2 & 3 & 4 & 5 & 6 & 7 \\ \text { Walker-like } & \text { K } & \text { G } & \text { G } & \text { X } & \text { X } & \text { K } & \text { T/S } \\ \text { EC MinD } & \text { K } & \text { G } & \text { G } & \text { V } & \text { G } & \text { K } & \text { T } \\ \text { P1 ParA } & \text { K } & \text { G } & \text { G } & \text { V } & \text { S } & \text { K } & \text { T } \\ \text { Aa Tadz } & \text { K } & \text { G } & \text { G } & \text { I } & \text { G } & \text { A } & \text { S } \\ \text { CC Tadz } & \text { K } & \text { G } & \text { G } & \text { V } & \text { G } & \text { A } & \text { S }\end{array}$

Fig. 8. Walker-like A boxes of proteins from the parA/minD superfamily.

Ec, Escherichia coli; P1, bacteriophage P1; Aa, Aggregatibacter actinomycetemcomitans;

Cc, Caulobacter crescentus

\subsection{Phenotypes of mutants altered in the atypical Walker-like A box of TadZ}

We wanted to know the effect of changing the residue in position 6 of the atypical Walkerlike A box from alanine in the TadZ protein of A. actinomycetemcomitans (AaTadZ) to the lysine residue of the canonical Walker-like A box. We used overlap extension PCR (Section 2.1) to change the codon in $\operatorname{tadZ}$ for residue 155 of the AaTadZ protein. We cloned the mutant gene into a plasmid vector and expressed it from the tac promoter, as described in Section 2.2. The mutant and wild-type proteins were found to be equally abundant in cells. After we introduced the plasmid into a tadZ mutant strain, we assayed three phenotypes of tad locus function: (1) change in colony morphology of a tadZ- mutant from smooth (the mutant phenotype) to rough (the wild-type phenotype), (2) autoaggregation of cells, and (3) formation of the tenacious biofilm. Whereas wild-type AaTadZ protein was completely functional in all three phenotypes, the A155K mutant protein was completely defective in all

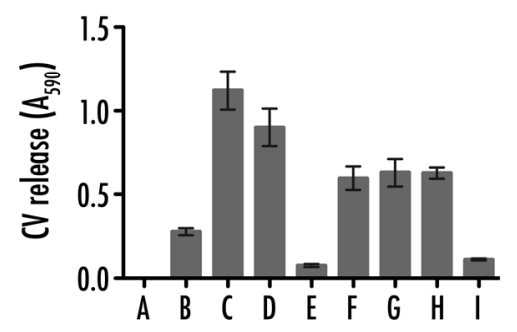

Fig. 9. Adherence of A. actinomycetemcomitans strains expressing the wild-type TadZ protein and mutants of TadZ.

Adherence was shown by the crystal violet $(\mathrm{CV})$ assay described in the text. The Y-axis is the same as in Fig. 6. A-D are controls: (A) media alone; (B) the tadZ- mutant strain with an empty vector plasmid; (C) a wild-type strain with vector; and (D) the tadZ- mutant strain with a plasmid carrying $t a d Z^{+}$. E-I are tadZ- strains expressing mutant TadZ proteins: (E) TadZ A155K, (F) TadZ K150R, (G) TadZ K150A, (H) TadZ S156T, and (I) TadZ S156A. The error bars show the calculated ranges of values. 
three phenotypes (see Fig. 9 for the adherence result). Therefore, the presence of alanine at position 6 of the Walker-like A box is essential for AaTadZ function.

We noticed that a common feature of the atypical Walker-like A boxes from other TadZ proteins was the absence of lysine at Walker-like box position 6, not the presence of alanine. The $t a d Z$ genes from various tad loci had codons for other amino acids at position 6 .

We confirmed this property for AaTadZ. When we mutated the tadZ gene to substitute a glycine, valine, asaparagine, or serine residue for alanine in the protein, all the mutant proteins were functional.

Even though it seemed that the absence of lysine was the primary requirement for the position 6 residue of the Walker-like A box of TadZ proteins, other residues of the Walkerlike A motif are conserved. This observation indicated that the other residues in the Walkerlike A boxes of TadZ proteins are important for function. To test this, we made mutants of AaTadZ in which the lysine residue at Walker-like A box position 1 (AaTadZ K150) was changed to arginine or alanine. Likewise, we made mutants at position 7 (AaTadZ S156) with threonine or alanine in place of the conserved serine residue. The mutant AaTadZ proteins did not allow wild-type biofilm formation (Fig. 9). Strains with the K150R, K150A, and the S156T mutants showed some biofilm formation; but it was reduced relative to wild type. The S156A mutant was completely unable to adhere. We concluded that the other residues of the atypical Walker-like A box of TadZ proteins are important to a function leading to tenacious adherence and biofilm formation.

The MinD proteins from E. coli (Hu \& Lutkenhaus, 1999; Raskin \& de Boer, 1999) and Neisseria gonorrhoea (Ramirez-Arcos et al., 2002), the ParA protein from plasmid pB171 of $E$. coli (Ebersbach \& Gerdes, 2002), and the ParA-like Soj protein of Bacillus subtilis (Marston \& Errington, 1999; Quisel et al., 1999) form polar foci and oscillate from pole to pole in the cell. The mobility of MinD-like and ParA-like proteins depends on their ATPase activity (Lutkenhaus \& Sundaramoorthy, 2003). Changing a residue in the Walker-like A box of ParA from pB171 (Ebersbach \& Gerdes, 2001), of the MinD protein from N. gonorrhoea (Ramirez-Arcos et al., 2002), or of Soj (Quisel et al., 1999) causes the protein to lose its mobility. Our TadZ-EGFP experiments have led us to suggest that TadZ positions the Tad secretion apparatus to a pole. Our hypothesis requires that TadZ does not oscillate. Maybe $\mathrm{AaTadZ}$ is a natural variant selected to allow it to remain stationary at a pole. The rest of the Walker-like A box may be needed to bind ATP, which was found in crystals of TadZ from Eubacterium rectale (Xu et al., 2012). The binding of ATP is not for the polar localization nor for dimer formation (see Sections 3.3 and 3.4 below). We note that ATP binding is necessary for the interaction of MinD with MinC and MinE (Hayashi et al., 2001; Hu et al., 2002), of Soj with Spo0J (Marston \& Errington, 1999; Quisel et al., 1999), and of bacteriophage P1 ParA with ParB (Bouet \& Funnell, 1999). Perhaps ATP is needed for TadZ to interact with one or more proteins of the Tad secretion apparatus.

\subsection{Mutants altered in the atypical Walker-like A box of TadZ localize properly}

We used PCR to amplify the $t a d Z$ genes that encoded the atypical Walker-like A box mutant proteins. The amplified mutant genes were used to make mutant TadZ-EGFP fusions, as described for the wild-type gene in Section 3. All of the mutant fusion proteins formed normal-appearing fluorescent foci at the old poles of cells. The absence of a defect in focus 
formation or location was particularly striking for the A155K and S156A mutants. A mutant TadZ protein with either allele was completely defective in the phenotypes we assayed (Fig. 9 and Section 3.2). In fact, the mutant fusions consistently showed a higher number of cells with polar foci than was seen with the wild-type TadZ-EGFP fusion. We do not understand the significance of the increase, but we concluded that the residues of the atypical Walkerlike A box are not needed for the polar localization of TadZ.

\subsection{Mutants altered in the atypical Walker-like A box of TadZ form dimers}

We used a bacterial reporter strain to indicate TadZ dimer formation in vivo (Hu et al., 2000). The basis of the reporter is the following. Bacteriophage $\lambda \mathrm{cI}$ protein represses the $\lambda p_{R}$ promoter. In the reporter strain, the $E$. coli lacZ gene, which encodes $\beta$-galactosidase, was fused to $p_{R}$. Because there is a convenient colorimetric assay for $\beta$-galactosidase, expression of lac $Z$ from the synthetic $p_{R}$-lacZ operon can be measured. The level of $\beta$-galactosidase is a function of $p_{R}$ activity and is, therefore, an indication of cI activity. cI protein is a dimer (Pabo et al., 1979). Each monomeric polypeptide has an N-terminal domain for DNA binding and a C-terminal domain for dimerization. If the C-terminal domain is removed, the N-terminal DNA-binding domain $(\lambda$ cIDB) is unable to function because it cannot form dimers. Fusing a polypeptide that can dimerize to $\lambda$ cIDB can make a functional repressor and reduce expression of $p_{R}-l a c Z$.

We created a chimeric gene that encoded a fusion of the coding regions for $\lambda$ cIDB and TadZ. The product of the fusion repressed $p_{R}-l a c Z$ and indicated that TadZ can form dimers (Table 4) (Perez-Cheeks et al., 2012). Knowing that TadZ can dimerize, we then asked if the atypical Walker-like A box mutants are proficient or defective in the dimerization activity (Perez-Cheeks et al., 2012). To do this, it was necessary to make chimeras of the coding region for $\lambda$ cIDB and the mutant tadZ genes. Each fusion protein was then tested in the reporter strain for the ability to repress $p_{R}$-lacZ. Each mutant fusion protein made a functional repressor that was as good as the fusion with wild-type TadZ (Table 4). We concluded that individual residues of the atypical Walker-like A box of TadZ are not needed for dimer formation.

\begin{tabular}{l|c} 
Protein & \% repression of $p_{R}$-lacZ \\
\hline Empty vector & $0.0 \pm 0.0$ \\
$\lambda$ cIDB & $9.7 \pm 4.0$ \\
$\lambda$ cI & $66.0 \pm 1.4$ \\
$\lambda$ cIDB-TadZ & $60.1 \pm 0.7$ \\
$\lambda$ cIDB-TadZ K150R & $49.0 \pm 1.9$ \\
$\lambda$ cIDB-TadZ K150A & $60.3 \pm 1.5$ \\
$\lambda$ cIDB-TadZ A155K & $49.7 \pm 2.1$ \\
$\lambda$ cIDB-TadZ S156T & $53.7 \pm 0.8$ \\
$\lambda$ cIDB-TadZ S156A & $57.6 \pm 4.2$ \\
\hline
\end{tabular}

Table 4. In vivo dimerization assay results for wild-type TadZ and mutant TadZ proteins. 


\section{Regulation of the tad locus}

Logic and evidence indicate that the expression of the tad locus genes in $A$. actinomycetemcomitans is controlled. The Tad- variants that spontaneously arise from $\mathrm{Tad}^{+}$ parents form larger colonies on solid medium, grow to a higher density in liquid medium, and have a shorter generation time than do their parents. We conclude that it is energetically expensive for a cell to make Flp pili. Therefore, it would be advantageous to the cell for tad locus expression to be regulated. One way to accomplish this is to control tad gene transcription.

There is evidence for transcriptional regulation of tad loci. P. aeruginosa has a tad locus, and it is regulated by the PprA-PprB two-component system (Bernard et al., 2009). The pprA and $p p r B$ genes map within a locus that has five contiguous, but divergent, transcriptional units. Four encode the tad genes and $p p r A$; the fifth encodes $p p r B$ only. After being activated by the histidine kinase (PprA), the response regulator (PprB) binds to the promoters and activates transcription of the tad genes. In another example, the expression of the tad genes (the flp operon) in the human pathogen Haemophilus ducreyi is affected by the CpxR-CpxA twocomponent system (Labandeira-Rey et al., 2010). Overexpression or constitutive activation of the response regulator $\mathrm{CpxR}$ causes repression of $f l p$ operon transcription and a reduction in the level of Flp1 protein. The authors suggested that activated CpxR directly represses transcription of the flp operon because CpxR bound to a target in the flp promoter region.

For A. actinomycetemcomitans, Kaplan et al. (2003) have shown the presence of probable nonadherent cells within a $\mathrm{Tad}^{+}$colony. They found sequestered, loosely packed, nonaggregating, and probably nonadherent cells in an adherent colony of $\mathrm{Tad}^{+}$cells. The authors suggested that transiently nonadherent cells are produced by the colony as part of a developmental pathway to expand the biofilm. Temporary nonadherence would allow cells to escape and seed new adherent colonies that are needed for the biofilm to spread. One possibility is that the nonadherent and non-autoaggregating cells lack Flp pili due to the inhibition of tad gene transcription. Indeed, there is a provocative 31-bp (base-pair) inverted repeat (IR) adjacent to the tad promoter (Fig. 3B) (G. Hovel-Miner, P. Planet, and D. Figurski, unpublished results). (The 31-bp IR has a spacer of $11 \mathrm{bp}$ flanked by 10-bp arms that are inverted complementary repeats of each other.) The IR is conserved in all six serotypes of $A$. actinomycetemcomitans, hinting that it is important to this organism. However, the function of the IR is currently unknown. Because the tad promoter is very strong (Kram et al., 2008), if the IR is a binding site for a protein that regulates transcription, it seems likely that the protein that binds IR would be a repressor that reduces tad transcription.

Our studies have indicated that tad locus_transcription is regulated by a termination cascade to maintain the correct stoichiometry of the tad gene products (Kram et al., 2008). We isolated three transcriptional terminators (T). T1 and T3 are factor-independent terminators, whereas $\mathrm{T} 2$ is a Rho-dependent terminator. RNA sizes and results from a lacZ transcriptional reporter indicated that $\mathrm{T} 1$ accounts for $\sim 99 \%$ termination and is located after the pilin gene, $f l p-1$, and before $f l p-2$. T2 is located between $t a d V$ and $r c p C$ and seems to terminate $\sim 36 \%$ of the remaining transcripts in our assay. T3 terminates transcription after tadG, i.e., at the end of the locus opposite the promoter. Rough RNA quantitation indicates that for every full-length tad transcript, there are $\sim 1.5 \mathrm{flp}-1 / \mathrm{flp}-2 / \mathrm{tadV}$ transcripts and $\sim 160$ flp-1 transcripts. This means that, assuming all tad mRNAs are translated with equal 
efficiencies, for every Tad secretion apparatus, there are about 50\% more TadV protease molecules to process the abundance of Flp1 prepilin protein.

\section{1 $\mathrm{Tad}^{+}$and $\mathrm{Tad}^{-}$bacteria make different biofilms}

Biofilm formation depends on adherence. Biofilms with different characteristics may indicate different modes of adherence. $\mathrm{Tad}^{+}$A. actinomycetemcomitans synthesize a pilibased, resilient biofilm. Tad- variants have been reported to form weak biofilms (Inoue et al., 2003). We noticed that cells of Tad- variants displayed adherence when the culture was handled gently (Fig. 10). Most or all of the biofilm of a Tad- strain is lost under the conditions we use to allow the biofilm of a $\mathrm{Tad}^{+}$strain to remain intact. Three-dimensional light microscopy showed that the biofilm of a Tad- strain is very different from the biofilm of its $\mathrm{Tad}^{+}$parent. Biofilms of $\mathrm{Tad}^{+}$strains showed distinct, tightly packed microcolonies of cells. In contrast, the biofilms of Tad- strains showed loosely packed cells in an extracellular matrix that stained readily with DAPI (4',6-diamidino-2-phenylindole, a fluorescent DNA stain). The biofilm of the Tad- strain showed cells in structures that were interpreted to be columns and mushroom shapes. We suggested that the Tad- biofilm resembles a "typical" biofilm.

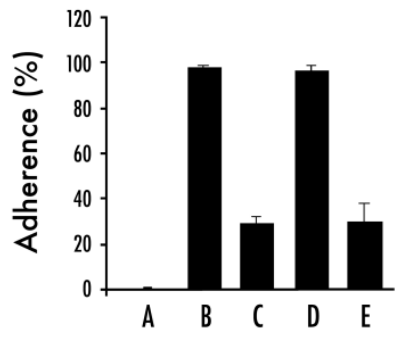

Fig. 10. Adherence of isogenic $\mathrm{Tad}^{+}$and Tad- strains of A. actinomycetemcomitans.

Shown are results from the crystal violet assay for adherence (see text) for two isogenic pairs of A. actinomycetemcomitans strains (B, C and D, E). A is a no-cells control. B and D are the $\mathrm{Tad}^{+}$parents: DF2200N (serotype a) and CU1000N (serotype f). The two spontaneous Tad(smooth-colony) variants are DF2261N (C) and CU1060N (E). The adherence of DF2200N is taken as $100 \%$. The error bars show the Standard Deviation calculated from the results of three experiments. Some adherence of the variants can be detected in conditions that favor the tenacious adherence of $\mathrm{Tad}^{+}$strains.

\subsection{Choosing proteins that may be needed for adherence to inert surfaces}

We sought to find a non-tad-locus protein needed for adherence in A. actinomycetemcomitans. We identified what we thought were prime candidates for adherence-essential proteins in $A$. actinomycetemcomitans (Table 5). We considered two-component systems because the two known examples of tad regulation by a non-tad determinant involve two-component systems. Only four two-component systems are predicted to be encoded by the genome of A. actinomycetemcomitans: ArcAB, CpxRA, NarPQ, and QseBC. We also selected two other proteins that we thought might be required for adherence: OxyR and PgaC. OxyR was chosen because Aggregatibacter aphrophilus, a bacterium closely related to $A$. 
actinomycetemcomitans, has a nearly identical tad locus (P. Planet, C. Sheth, and D. Figurski, unpublished results; Di Bonaventura et al., 2009). A. aphrophilus makes more Flp pili in higher oxygen than in lower (S. Kachlany, C. Sheth, and D. Figurski, unpublished results). Therefore, we selected OxyR, a transcriptional activator that responds to oxygen (Bauer et al., 1999), because the tad genes in this bacterium appear to respond to the presence of oxygen. In addition, there is evidence that OxyR induces the adhesin apiA in $A$. actinomycetemcomitans (Ramsey \& Whiteley, 2009). PGA (poly- $\beta-1,6-N$-acetyl-Dglucosamine) is an extracellular polymer that is synthesized by A. actinomycetemcomitans and is associated with the biofilm of $\mathrm{Tad}^{+}$cells (Izano et al., 2008). We considered PgaC, an enzyme in the pathway for PGA synthesis, to be a candidate for a protein that affects a biofilm in A. actinomycetemcomitans.

\begin{tabular}{l|l} 
Gene & Function \\
\hline $\operatorname{arcB}$ & Two-component sensor kinase; response to anaerobic and aerobic stress \\
$c p x R$ & Two-component response regulator; response to outer membrane stress \\
$n a r P$ & Two-component response regulator; response to nitrate and nitrite stress \\
$q s e B$ & Two-component response regulator; response to quorum sensing \\
oxyR & Transcriptional regulator; response to redox stress \\
$p g a C$ & Integral membrane glycosyltransferase; required for PGA biosynthesis \\
\hline
\end{tabular}

Table 5. Selected putative regulators of adherence by A. actinomycetemcomitans.

\subsection{Mutagenesis of possible adherence-required genes by allelic exchange}

We constructed a series of six mutant $\mathrm{Tad}^{+}$strains and six mutant Tad- strains. Each strain had a mutation in one of our six candidates genes (Section 4.2). We asked if any of the mutants was defective in the strain's biofilm. The mutant strains were made by allelic exchange, i.e., the mutant gene was substituted for the wild-type gene in the chromosome (Fig. 11). In allelic exchange, the mutant gene is marked (usually with an antibiotic resistance gene) and introduced into a wild-type strain. Homologous recombination allows the mutant gene and its marker to replace the wild-type gene in the chromosome. If the gene is not essential for viability, strains in which the exchange has taken place can be selected because they are able to grow in the presence of the marker antibiotic. Strains that have not done the exchange will not grow in the presence of the antibiotic because the introduced DNA cannot replicate.

We used plasmid pMB78 (Bhattacharjee et al., 2007), a "suicide" plasmid, i.e., one that can replicate or be maintained in one host and not in another. Plasmid pMB78 can be maintained in E. coli, but not in A. actinomycetemcomitans. The wild-type gene was amplified from the chromosome of A. actinomycetemcomitans and cloned into pMB78 in E. coli. To construct the mutant allele, the internal part of the wild-type gene was deleted and replaced with the gene for kanamycin resistance. Plasmid pMB78 has the uptake signal sequence (USS) needed for transformation of A. actinomycetemcomitans. After transformation and recombination, the recombinants (i.e., the mutant strains) were selected by their growth on medium containing kanamycin. The presence of the mutant alleles was confirmed by PCR and gel electrophoresis (Fig. 12A). 


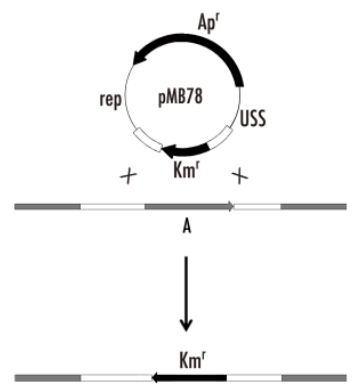

Fig. 11. Schematic for allelic exchange.

pMB78 is an ampicillin resistance (Apr)-encoding suicide plasmid that cannot be maintained in A. actinomcetemcomitans (see text) (Bhattacharjee et al., 2007). The wild-type gene is cloned and mutated by insertion of DNA encoding (in this example) kanamycin resistance $\left(\mathrm{Km}^{\mathrm{r}}\right)$. After recombination (" $X$ ") in the homologous regions (white boxes), the mutated gene replaces the wild-type gene in the chromosome. The mutant strains (recombinants) can grow in the presence of kanamycin. "rep" is "replication region"; "USS," "uptake signal sequence," which is needed for transformation of A. actinomycetemcomitans (Thomson et al., 1999).

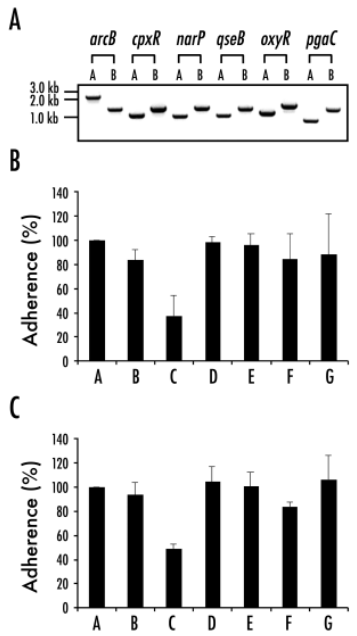

Fig. 12. Directed mutations and their effects on the adherence of $\mathrm{Tad}^{+}$and Tad- strains of $A$. actinomycetemcomitans.

Panel A shows agarose gel electrophoresis of PCR products of the wild-type gene and the mutant gene after recombination for each of the six possible regulators of adherence. Panels $B$ and $C$ show the results of the crystal violet assay for adherence (see text) of the isogenic $\mathrm{Tad}^{+}$and Tad- strains, respectively. In Panels B and C, adherence of the parent strain (A) is normalized to $100 \%$. The mutant strains are defective in $\operatorname{arcB}(\mathrm{B}), \operatorname{cpxR}(\mathrm{C}), \operatorname{nar} P(\mathrm{D})$, qseB $(\mathrm{E})$, $\operatorname{oxyR}(\mathrm{F})$, and $\operatorname{pgaC}(\mathrm{G})$. 
Adherence of the $\mathrm{Tad}^{+}$(Fig. 12B) and Tad- (Fig. 12C) mutants was quantified by the crystal violet assay (Section 2.2). One mutation, $\Delta c p x R:: \mathrm{Km}^{\mathrm{r}}$ ("::" indicates "insertion of"), consistently caused a significant decrease in adherence (relative to the wild-type strain) for both the $\mathrm{Tad}^{+}$and $\mathrm{Tad}^{-}$strains (Fig. $12 \mathrm{~B}$ and C, respectively). This result showed that the $\triangle c p x R:: \mathrm{Km}^{\mathrm{r}}$ mutation affected adherence in both types of strains. An important confirmation was to ask if adding the intact $c p x R^{+}$gene in trans restored adherence to the mutant strains (genetic complementation). If it did, then we could conclude that the defect in the mutant strain was the result of not having a functional CpxR protein. The $c p x R^{+}$gene was added in trans as a cloned gene on a plasmid that replicates in A. actinomycetemcomitans. We were surprised to learn that $c p x R^{+}$in trans restored adherence to the Tad- strain (Fig. 13A), but not to the $\mathrm{Tad}^{+}$strain (Fig. 13B). For the $\mathrm{Tad}^{+}$strain, genetic complementation occurred only when the complete cpxRA operon was added in trans, indicating the need for CpxA in Tad ${ }^{+}$ adherence (Fig. 13B). More needs to be done to make this a solid conclusion. However, we can conclude that both types of adherence in A. actinomycetemcomitans respond to a function encoded by the cpxRA operon. We wish to point out that adherence in $A$. actinomycetemcomitans is positively regulated by the cpxRA operon, and we do not know whether it functions directly or indirectly to regulate adherence. In contrast, the activation of CpxR in H. ducreyi leads the molecule to bind to the tad (flp operon) promoter as a negative regulator (Labandeira-Rey et al., 2010).

\section{A strategy for making precise genomic deletions: the new Vector Excision (VEX) method}

In Section 4.3, allelic exchange was described as a method of exchanging a wild-type segment of the chromosome with a mutated segment. In the examples given, a single gene was mutated. Allelic exchange can also be used for more than one gene. However, because the technique usually relies on standard molecular cloning methods, allelic exchange often becomes more difficult with larger segments. (See the chapter by Gerlach et al. for a discussion of recombineering - a method of cloning that overcomes the limitation caused by the locations of restriction endonuclease cleavage sites.) Another problem can be caused by the insertion of the marker, which can affect the expression of downstream genes. A method based on site-specific recombination has been developed to remove the marker (Datsenko and Wanner, 2000).

We have developed a straightforward method for making chromosomal deletions that can be any size [one bp to several kb (kilobase pairs)] and do not affect the expression of downstream genes. The strategy is based on the Vector Excision (VEX) method that we developed (Ayres et al., 1993). With VEX, the deleted portion can also be "captured" on a self-transmissible, broad-host-range plasmid. After transfer to another bacterium, the expression and/or functions of the captured genes can be assessed in a different host (see the chapter by Wilson et al.).

The new VEX strategy is illustrated here as a deletion $(\sim 11 \mathrm{~kb})$ of all the tad genes of $A$. actinomycetemcomitans (Fig. 14). A "double cointegrate" is generated by two cycles of homologous recombination. Essentially the double cointegrate is formed by two successive allelic exchanges (Section 4.3). The plasmid suicide vector we used was based on pBBR1MCS (Kovach et al., 1994), which replicates in E. coli, but not in $A$. 
actinomycetemcomitans. The plasmid can be mobilized into both species by conjugation, and the USS required for transformation of A. actinomycetemcomitans was added along with the gene for ampicillin resistance by recombineering. Thus, the vector can be introduced into $A$. actinomycetemcomitans either by transformation (as was done here) or by conjugative mobilization (crucial for strains that transform poorly or not at all).
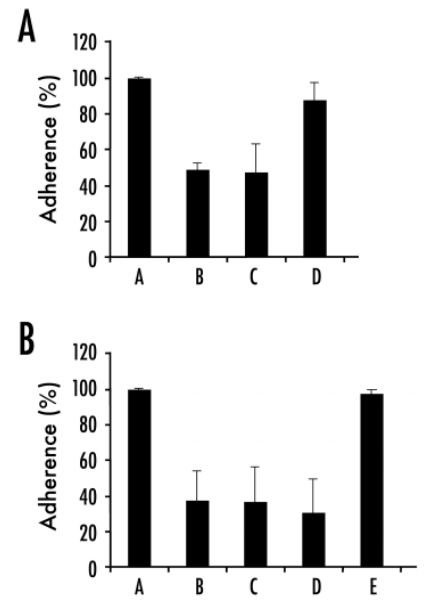

Fig. 13. Genetic complementation of the adherence defect from the $c p x R$ - mutation in isogenic $\mathrm{Tad}^{-}$and $\mathrm{Tad}^{+}$strains of A. actinomycetemcomitans.

Shown are crystal violet assays for adherence (see text). Panel A is the Tad- strain; panel B is the $\mathrm{Tad}^{+}$strain. Column A in panel A is the smooth-colony variant with the wild-type cpxR gene. Its adherence is normalized to $100 \%$. Column B is the cpxR- mutant strain; column C, the $c p x R^{-}$mutant strain with the empty plasmid vector; and column $\mathrm{D}$, the $c p x R^{-}$mutant strain with the $c p x R^{+}$plasmid. Column A in panel B is the $\mathrm{Tad}^{+}$parent. B-D are analogous to those in Panel A, but with the $\mathrm{Tad}^{+}$stain. Column $\mathrm{E}$ is the $c p x \mathrm{R}^{-}$mutant strain with a cpxRA+ plasmid. Note that complementation of the $c p x R$ - mutation in the $\mathrm{Tad}^{+}$strain requires cp $x R A^{+}$in trans, whereas cpx $R^{+}$in trans is sufficient for complementation of the $c p x R^{-}$ mutation in the Tad- strain.

The purpose of the two homologous recombination events is to integrate two directly repeated $\operatorname{lox} P$ sites. The homology cassette determines where the loxP sequence is inserted into the chromosome. In our experiment, one loxP sequence was inserted in the flp-1 gene; and the other, in tadG. The "left" (in Fig. 14) loxP sequence was marked by the aac $\mathrm{C} 1$ gene for gentamicin resistance. The "right" loxP sequence was marked by aadA, the gene for spectinomycin resistance. Each loxP cassette was formed by cloning three fragments into the multiple cloning site (MCS) of the vector: (1) homology region I (HRI), (2) loxP and aacC1 or $a a d A$, and (3) homology region II (HRII). The fragments were generated by PCR. The 34-bp $\operatorname{lox} P$ sequence was added to the appropriate primer for fragment (2). Fragments were 
A
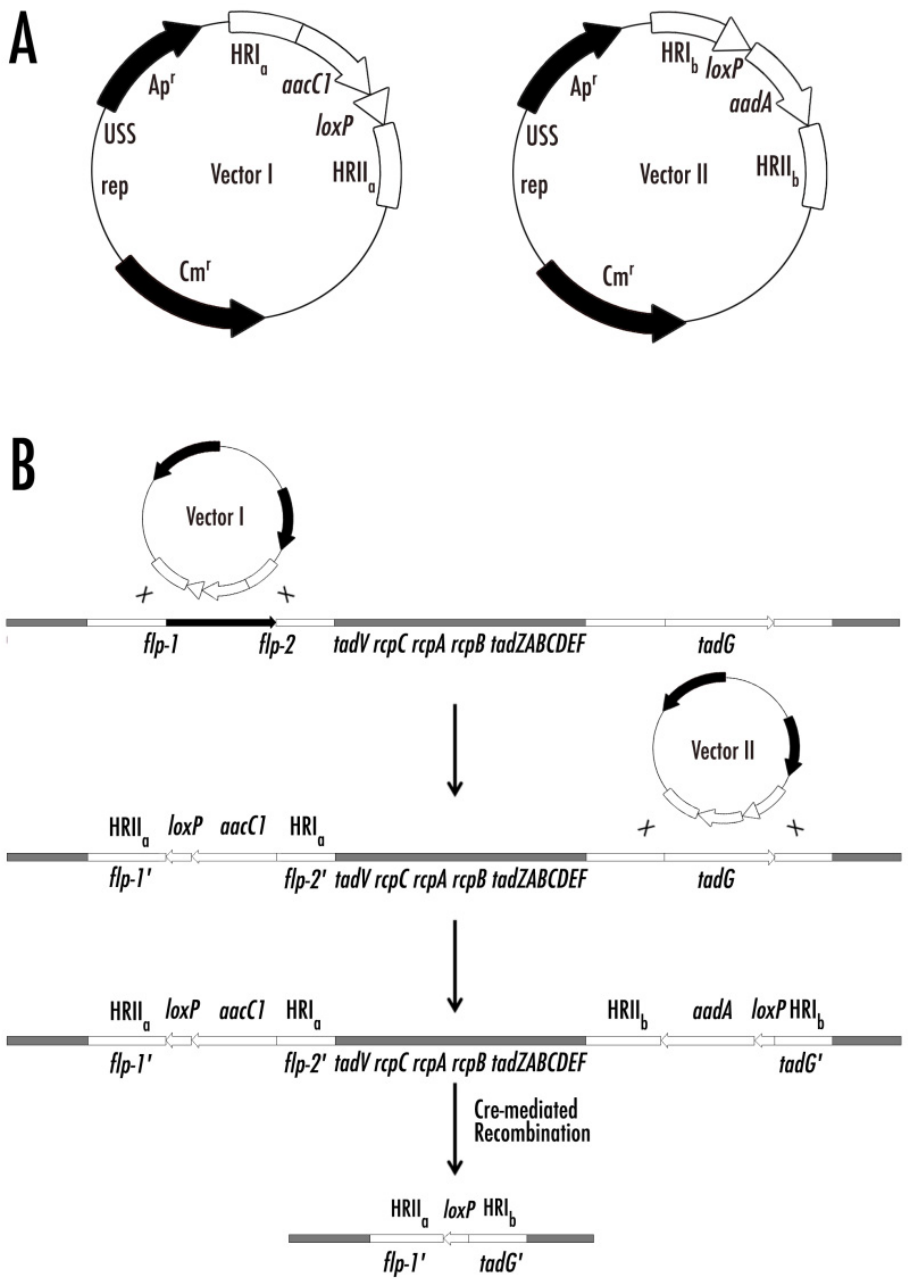

Fig. 14. Schematic for the "new VEX" method of making precise genomic deletions. Panel A shows the two plasmids that will be used in the construction of the double cointegrate. Vectors I and II are suicide plasmids that can replicate in E. coli, but not in A. actinomycetemcomitans. They are made from the same plasmid. The symbols and abbreviations are the following: the loxP cassettes (with cloned fragments) are in white; "Apr," ampicillin resistance; "Cmr," chloramphenicol resistance; "rep," replicon; "USS," uptake signal sequence for transformation of A. actinomycetemcomitans; "HR," homology region; "aacC1," the gene for gentamicin resistance; "aadA," the gene for spectinomycin resistance; and "loxP," a 34-bp sequence that is the target for Cre-mediated site-specific recombination. Panel B depicts the two homologous recombination events and resolution by the Cre recombinase to delete the genes of the tad locus in A. actinomycetemcomitans. See the text for details. 
cloned with the following restriction endonucleases: $\mathrm{Xba \textrm {I }}$ and BamHI for fragment (1), BamHI and SalI for fragment (2), and SalI and KpnI for fragment (3). The site-specific recombinase Cre binds to a loxP sequence (Sternberg and Hoess, 1983), forms a synapse with another Cre-bound loxP sequence, and catalyzes recombination between $\operatorname{lox} P$ sequences. Cre-mediated recombination is very efficient (>95\%). Directly repeated loxP sites cause the intervening DNA to cyclize and be deleted by the action of Cre. Inverted repeats of loxP sites lead to inversion of the intervening DNA. Cre was supplied to the double cointegrate by conjugation with a cre-encoding plasmid.

After the Cre-mediated deletion (resolution) (Fig. 15), a single loxP sequence is left in place of the deleted DNA (Fig. 14). In the $f l p-1$ to $\operatorname{tadG}$ deletion, $11 \mathrm{~kb}$ of DNA was removed, whereas one 34-bp loxP sequence was inserted. Our results show that a double loxPcontaining homology cassette with antibiotic resistance genes blocks transcription of a downstream gene. In contrast, the single loxP sequence present after resolution allows transcription and expression of the downstream gene (T. McConville and D. Figurski, unpublished results).

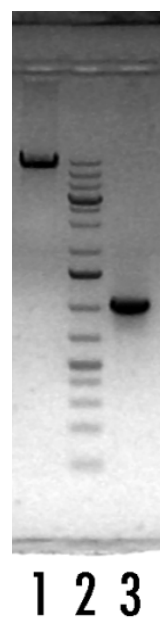

Fig. 15. Deletion of the tad locus of A. actinomycetemcomitans.

Shown is an agarose gel of PCR products that show the wild-type tad locus (1) and a genomic deletion of $\sim 11 \mathrm{~kb}$ that removes all the genes of the tad locus (3). The deletion was made using the new VEX strategy shown in Fig. 14. Lane 2 shows DNA markers of different molecular weights.

\section{Conclusion}

The 14-gene tad locus of A. actinomycetemcomitans is needed for the synthesis and secretion of Flp pili. The ability of A. actinomycetemcomitans to cause periodontal disease depends on Flp pili-mediated tenacious adherence in the oral cavity. (Flp pili likely mediate colonization of 
teeth.) We think we may understand the function of Flp pili in the etiology of oral disease caused by A. actinomycetemcomitans. However, tad loci occur in nearly $40 \%$ of prokaryotes. We have suggested that tad loci help prokaryotes colonize a niche (Kachlany et al., 2001a). For $A$. actinomycetemcomitans, a major niche is known to be the oral cavity. Tenacious adherence may be a property of $A$. actinomycetemcomitans because it must be able to colonize in the presence of extensive normal flora. Therefore, the strong phenotype of tenacious adherence may be a special property of the tad locus of A. actinomycetemcomitans. The strong phenotype allowed us to discover the tad genes. The phenotypes may be important, but more subtle, in other organisms.

Several proteins are unique products of tad loci. Such proteins may be good targets for therapeutic drugs. For example, tadZ is present in every tad locus. Our genetic studies in A. actinomycetemcomitans have shown that the $t a d Z$ gene is required for the function of its tad locus. We believe it is likely to be important in all tad loci. Therefore, a drug specific for TadZ protein might inactivate all tad loci. We have shown that the tad locus of $A$. actinomycetemcomitans is required for colonization of the mouth and periodontal disease. If we are correct that tad loci in other prokaryotes are needed to colonize their specific niches, inhibiting TadZ to inactivate tad loci may be useful in preventing and/or minimizing some diseases. Such a drug that targets a non-essential colonization factor should also be largely refractory to the selective pressure for the emergence of resistant strains.

Genetic studies of tad loci and the development of new genetic tools are helping us to determine and understand (1) the functions of the individual tad genes, (2) whether and how the various proteins encoded by a tad locus act to colonize a specific niche, and (3) the importance of the tad genes for A. actinomycetemcomitans and for other prokaryotes.

\section{Acknowledgments}

We are grateful to David Furgang, Scott Kachlany, Jeff Kaplan, Mari Karched, Paul Planet, Helen Schreiner, Kabilan Velliyagounder, James Wilson, and Gang Yue for discussions and/or technical help. We appreciate the efforts of Oliver Jovanovic on the figures. The work was funded by grants from the National Institutes of Health (NIH), USA. Additional NIH funding supported B.A.P., V.W.G., and K.E.K.

\section{References}

Ayres, E.K., Thomson, V.J., Merino, G., Balderes, D. \& Figurski, D.H. (1993). Precise deletions in large bacterial genomes by vector-mediated excision (VEX). The trfA gene of promiscuous plasmid RK2 is essential for replication in several gramnegative hosts, J. Mol. Biol. 230, 174-185.

Bauer, C.E., Elsen, S. \& Bird, T.H. (1999). Mechanisms for redox control of gene expression, Annu. Rev. Microbiol. 53, 495-523.

Bernard, C.S., Bordi, C., Termine, E., Filloux, A. \& de Bentzmann, S. (2009). Organization and PprB-dependent control of the Pseudomonas aeruginosa tad Locus, involved in Flp pilus biology, J. Bacteriol. 191, 1961-1973. 
Bhattacharjee, M.K., Kachlany, S.C., Fine, D.H. \& Figurski, D.H. (2001). Nonspecific adherence and fibril biogenesis by Actinobacillus actinomycetemcomitans: TadA protein is an ATPase, J. Bacteriol. 183, 5927-5936.

Bhattacharjee, M.K., Fine, D.H. \& Figurski, D.H. (2007). tfoX (sxy)-dependent transformation of Aggregatibacter (Actinobacillus) actinomycetemcomitans, Gene 399, 53-64.

Bouet, J.Y. \& Funnell, B.E. (1999). P1 ParA interacts with the P1 partition complex at parS and an ATP-ADP switch controls ParA activities, EMBO J. 18, 1415-1424.

Clock, S.A., Planet, P.J., Perez, B.A. \& Figurski, D.H. (2008). Outer membrane components of the Tad (tight adherence) secreton of Aggregatibacter actinomycetemcomitans, J. Bacteriol. 190, 980-990.

Datsenko, K.A. \& Wanner, B.L. (2000). One-step inactivation of chromosomal genes in Escherichia coli K-12 using PCR products, Proc. Natl. Acad. Sci. USA 97(12):6640-5.

de Bentzmann, S., Aurouze, M., Ball, G. \& Filloux, A. (2006). FppA, a novel Pseudomonas aeruginosa prepilin peptidase involved in assembly of type IVb pili, J. Bacteriol. 188, 4851-4860.

De Rycke, J. \& Oswald, E. (2001). Cytolethal distending toxin (CDT): a bacterial weapon to control host cell proliferation? FEMS Microbiol. Lett. 203, 141-148.

Di Bonaventura, M.P., DeSalle, R., Pop, M., Nagarajan, N., Figurski, D.H., Fine, D.H., Kaplan, J.B. \& Planet, P.J. (2009). Complete genome sequence of Aggregatibacter (Haemophilus) aphrophilus NJ8700, J. Bacteriol. 191, 4693-4694.

DiFranco, K.M., Gupta, A., Galusha, L.E., Perez, J., Nguyen, T.V., Fineza, C.D. \& Kachlany, S.C. (2012). Leukotoxin (Leukothera $\left.{ }^{\circledR}\right)$ targets active leukocyte function antigen-1 (LFA-1) protein and triggers a lysosomal mediated cell death pathway, J. Biol. Chem. 287, 17618-17627.

Ebersbach, G. \& Gerdes, K. (2001). The double par locus of virulence factor pB171: DNA segregation is correlated with oscillation of ParA, Proc. Natl. Acad. Sci. USA 98, 15078-15083

Fine, D.H., Furgang, D., Kaplan, J., Charlesworth, J. \& Figurski. D.H. (1999a). Tenacious adhesion of Actinobacillus actinomycetemcomitans strain CU1000 to salivary-coated hydroxyapatite. Arch. Oral. Biol. 44, 1063-1076.

Fine, D.H., Furgang, D., Schreiner, H.C., Goncharoff, P., Charlesworth, J., Ghazwan, G., Fitzgerald-Bocarsly, P. \& Figurski, D.H. (1999b). Phenotypic variation in Actinobacillus actinomycetemcomitans during laboratory growth: implications for virulence, Microbiology 145, 1335-1347.

Fine, D.H., Goncharoff, P., Schreiner, H., Chang, K.M., Furgang, D. \& Figurski, D. (2001). Colonization and persistence of rough and smooth colony variants of Actinobacillus actinomycetemcomitans in the mouths of rats, Arch. Oral Biol. 46, 1065-1078.

Fine, D.H., Velliyagounder, K., Furgang, D. \& Kaplan, J.B. (2005) The Actinobacillus actinomycetemcomitans autotransporter adhesin Aae exhibits specificity for buccal epithelial cells from humans and old world primates, Infect. Immun. 73, 1947-1953.

Fine, D.H., Kaplan, J.B., Kachlany, S.C. \& Schreiner, H.C. (2006). How we got attached to Actinobacillus actinomycetemcomitans: a model for infectious diseases, Periodont. 2000 42, 114-157. 
Fine, D.H., Kaplan, J.B., Furgang, D., Karched, M., Velliyagounder, K. \& Yue, G. (2010). Mapping the epithelial-cell-binding domain of the Aggregatibacter actinomycetemcomitans autotransporter adhesin Aae, Microbiology 156, 3412-3420.

Fives-Taylor, P.M., Meyer, D.H., Mintz, K.P. \& Brissette, C. (1999). Virulence factors of Actinobacillus actinomycetemcomitans, Periodontol. 2000 20, 136-167.

Fong, K.P., Pacheco, C.M., Otis, L.L., Baranwal, S., Kieba, I.R., Harrison, G., Hersh, E.V., Boesze-Battaglia, K. \& Lally, E.T. (2006). Actinobacillus actinomycetemcomitans leukotoxin requires lipid microdomains for target cell cytotoxicity, Cell Microbiol. 8, 1753-1767.

Fuller, T.E., Kennedy, M.J. \& Lowery, D.E. (2000). Identification of Pasteurella multocida virulence genes in a septicemic mouse model using signature-tagged mutagenesis, Microb. Pathog. 29, 25-38.

Genco, R.J., Christersson, L.A. \& Zambon, J.J. (1986). Juvenile periodontitis, Int. Dent. J. 36, 168-176.

Giltner, C.L., Nguyen, Y. \& Burrows, L.L. (2012). Type IV Pilin Proteins: Versatile Molecular Modules, Microbiol. Mol. Biol. Rev. 76, 740-772.

Haase, E.M., Zmuda, J.L. \& Scannapieco, F.A. (1999). Identification and molecular analysis of rough-colony-specific outer membrane proteins of Actinobacillus actinomycetemcomitans, Infect. Immun. 67, 2901-2908

Haase, E.M., Stream, J.O. \& Scannapieco, F.A. (2003). Transcriptional analysis of the 5' terminus of the flp fimbrial gene cluster from Actinobacillus actinomycetemcomitans, Microbiology 149(Pt 1), 205-215.

Hayashi, I., Oyama, T. \& Morikawa, K. (2001). Structural and functional studies of MinD ATPase: implications for the molecular recognition of the bacterial cell division apparatus, EMBO J. 20, 1819-1828.

Henderson, B., Wilson, M., Sharp, L. \& Ward, J.M. (2002). Actinobacillus actinomycetemcomitans, J. Med. Microbiol. 51, 1013-1020.

Henderson, B., Nair, S.P., Ward, J.M. \& Wilson, M. (2003). Molecular pathogenicity of the oral opportunistic pathogen Actinobacillus actinomycetemcomitans, Annu. Rev. Microbiol. 57, 29-55.

Henderson B., Ward J.M. \& Ready D. (2010). Aggregatibacter (Actinobacillus) actinomycetemcomitans: a triple A* periodontopathogen? Periodontol. 2000 54, 78-105.

Ho, S.N., Hunt, H.D., Horton, R.M., Pullen, J.K. \& Pease, L.R. (1989). Site-directed mutagenesis by overlap extension using the polymerase chain reaction, Gene 77, 5159.

Hu, J.C., Kornacker, M.G. \& Hochschild, A. (2000). Escherichia coli one- and two-hybrid systems for the analysis and identification of protein-protein interactions, Methods 20, 80-94.

Hu, Z. \& Lutkenhaus, J. (1999). Topological regulation of cell division in Escherichia coli involves rapid pole to pole oscillation of the division inhibitor MinC under the control of MinD and MinE, Mol. Microbiol. 34, 82-90.

$\mathrm{Hu}$, Z., Gogol, E.P. \& Lutkenhaus, J. (2002). Dynamic assembly of MinD on phospholipid vesicles regulated by ATP and MinE, Proc. Natl. Acad. Sci. USA 99, 6761-6766. 
Inoue, T., Tanimoto, I., Ohta, H., Kato, K., Murayama, Y. \& Fukui, K. (1998). Molecular characterization of low-molecular-weight component protein, Flp, in Actinobacillus actinomycetemcomitans fimbriae, Microbiol. Immunol. 42, 253-258.

Inoue, T., Ohta, H., Tanimoto, I., Shingaki, R. \& Fukui, K. (2000). Heterogeneous posttranslational modification of Actinobacillus actinomycetemcomitans fimbrillin, Microbiol. Immunol. 44, 715-718.

Izano, E.A., Sadovskaya, I., Wang, H., Vinogradov, E., Ragunath, C., Ramasubbu, N., Jabbouri, S., Perry, M.B. \& Kaplan, J.B. (2008). Poly-N-acetylglucosamine mediates biofilm formation and detergent resistance in Aggregatibacter actinomycetemcomitans, Microb. Pathog. 44, 52-60.

Jiang, X., Ruiz, T. \& Mintz, K.P. (2012). Characterization of the secretion pathway of the collagen adhesin EmaA of Aggregatibacter actinomycetemcomitans, Mol Oral Microbiol. 27, 382-396.

Kachlany, S.C., Planet, P.J., Bhattacharjee, M.K., Kollia, E., DeSalle, R., Fine, D.H. \& Figurski, D.H. (2000). Nonspecific adherence by Actinobacillus actinomycetemcomitans requires genes widespread in bacteria and archaea. J. Bacteriol. 182, 6169-6176.

Kachlany, S.C., Planet, P.J., DeSalle, R., Fine, D.H., \& Figurski, D.H. (2001a). Genes for tight adherence of Actinobacillus actinomycetemcomitans: from plaque to plague to pond scum. Trends Microbiol. 9, 429-437.

Kachlany, S.C., Planet, P.J., DeSalle, R., Fine, D.H., Figurski, D.H. \& Kaplan, J.B. (2001b). flp1 , first representative of a new pilin gene subfamily, is required for nonspecific adherence of Actinobacillus actinomycetemcomitans, Mol. Microbiol. 40, 542-554.

Kachlany, S.C. (2010). Aggregatibacter actinomycetemcomitans leukotoxin: from threat to therapy, J. Dent. Res. 89, 561-570.

Kaplan, J.B., Meyenhofer, M.F. \& Fine, D.H. (2003). Biofilm growth and detachment of Actinobacillus actinomycetemcomitans, J. Bacteriol. 185, 1399-1404.

Kelk, P., Johansson, A., Claesson, R., Hänström, L. \& Kalfas, S. (2003). Caspase 1 involvement in human monocyte lysis induced by Actinobacillus actinomycetemcomitans leukotoxin, Infect. Immun. 71, 4448-4455.

Kelk, P., Abd, H., Claesson, R., Sandström, G., Sjöstedt, A. \& Johansson, A. (2011). Cellular and molecular response of human macrophages exposed to Aggregatibacter actinomycetemcomitans leukotoxin. Cell Death Dis. 2, e126.

Kovach, M.E., Phillips, R.W., Elzer, P.H., Roop II, R.M. \& Peterson, K.M. (1994). pBBR1MCS: a broad-host-range cloning vector, Biotechniques 16, 800-802.

Kram, K.E., Hovel-Miner, G.A., Tomich, M. \& Figurski, D.H. (2008). Transcriptional regulation of the tad locus in Aggregatibacter actinomycetemcomitans: a termination cascade, J. Bacteriol. 190, 3859-3868.

Komatsuzawa, H., Asakawa, R., Kawai, T., Ochiai, K., Fujiwara, T., Taubman, M.A., Ohara, M., Kurihara, H. \& Sugai, M. (2002). Identification of six major outer membrane proteins from Actinobacillus actinomycetemcomitans, Gene 288, 195-201.

Labandeira-Rey, M., Brautigam, C.A. \& Hansen, E.J. (2010). Characterization of the CpxRA regulon in Haemophilus ducreyi, Infect. Immun. 78, 4779-4791.

Lally, E.T., Hill, R.B., Kieba, I.R. \& Korostoff, J. (1999). The interaction between RTX toxins and target cells, Trends Microbiol. 7, 356-361. 
Löe, H. \& Brown, L.J. (1991). Early onset periodontitis in the United States of America, J. Periodontol. 62, 608-616.

Lutkenhaus, J. \& Sundaramoorthy, M. (2003). MinD and role of the deviant Walker A motif, dimerization and membrane binding in oscillation, Mol. Microbiol. 48, 295-303.

Marston, A.L. \& Errington, J. (1999). Dynamic movement of the ParA-like Soj protein of B. subtilis and its dual role in nucleoid organization and developmental regulation, Mol. Cell 4, 673-682.

Mintz, K.P. (2004). Identification of an extracellular matrix protein adhesin, EmaA, which mediates the adhesion of Actinobacillus actinomycetemcomitans to collagen. Microbiology 150, 2677-2688.

O'Toole, G. A. \& Kolter. R. (1998). Initiation of biofilm formation in Pseudomonas fluorescens WCS365 proceeds via multiple, convergent signalling pathways: a genetic analysis, Mol. Microbiol. 28, 449-461.

Pabo, C.O., Sauer, R.T., Sturtevant, J.M. \& Ptashne, M. (1979). The lambda repressor contains two domains, Proc. Natl. Acad. Sci. USA 76, 1608-1612.

Paturel, L., Casalta, J.P., Habib, G., Nezri, M. \& Raoult, D. (2004). Actinobacillus actinomycetemcomitans endocarditis, Clin. Microbiol. Infect. 10, 98-118.

Perez, B.A., Planet, P.J., Kachlany, S.C., Tomich, M., Fine, D.H. \& Figurski, D.H. (2006). Genetic analysis of the requirement for $f l p-2, \operatorname{tadV}$, and $r c p B$ in Actinobacillus actinomycetemcomitans biofilm formation. J. Bacteriol. 188, 6361-6375.

Perez-Cheeks, B.A., Planet, P.J., Sarkar, I.N., Clock, S.A., Xu, Q. \& Figurski, D.H. (2012). The product of $t a d Z$, a new member of the $\operatorname{par} A / \min D$ superfamily, localizes to a pole in Aggregatibacter actinomycetemcomitans, Mol. Microbiol. 83, 694-711.

Pickett, C.L. \& Whitehouse, C.A. (1999). The cytolethal distending toxin family, Trends Microbiol. 7, 292-297.

Planet, P.J., Kachlany, S.C., Fine, D.H., DeSalle, R., and Figurski, D.H. (2003) The Widespread Colonization Island (WCI) of Actinobacillus actinomycetemcomitans, Nat. Genet. 34, 193-198.

Quisel, J.D., Lin, D.C. \& Grossman, A.D. (1999). Control of development by altered localization of a transcription factor in B. subtilis, Mol. Cell 4, 665-672.

Rahamat-Langendoen J.C., van Vonderen, M.G.A., Engström, L.J., Manson, W.L., van Winkelhoff, A.J. \& Mooi-Kokenberg, E.A.N.M. (2011). Brain abscess associated with Aggregatibacter actinomycetemcomitans: case report and review of literature, J. Clin. Periodontol. 38, 702-706.

Ramirez-Arcos, S., Szeto, J., Dillon, J-A.R. \& Margolin, W. (2002). Conservation of dynamic localization among MinD and MinE orthologues: oscillation of Neisseria gonorrhoeae proteins in Escherichia coli, Mol. Microbiol. 46, 493-504.

Ramsey, M.M. \& Whiteley, M. (2009). Polymicrobial interactions stimulate resistance to host innate immunity through metabolite perception, Proc. Natl. Acad. Sci. USA 106, 1578-1583.

Raskin, D.M., \& de Boer, P.A. (1999). Rapid pole-to-pole oscillation of a protein required for directing division to the middle of Escherichia coli, Proc. Natl. Acad. Sci. USA 96, 4971-4976. 
Rose, J.E., Meyer, D.H. \& Fives-Taylor, P.M. (2003). Aae, an autotransporter involved in adhesion of Actinobacillus actinomycetemcomitans to epithelial cells. Infect. Immun. 71, 2384-2393.

Rylev, M. \&, Kilian, M. (2008). Prevalence and distribution of principal periodontal pathogens worldwide, J. Clin. Periodontol. 35, 346-361.

Schreiner, H.C., Sinatra, K., Kaplan, J.B., Furgang, D., Kachlany, S.C., Planet, P.J., Perez, B.A., Figurski, D.H. \& Fine, D.H. (2003). Tight adherence genes of Actinobacillus actinomycetemcomitans are required for virulence in a rat model, Proc. Natl. Acad. Sci. USA 12, 7295-7300.

Slots, J. \& Ting, M. (1999). Actinobacillus actinomycetemcomitans and Porphyromonas gingivalis in human periodontal disease: occurrence and treatment, Periodontol. 2000 20, 82121.

Spinola, S. M., Fortney, K.R., Katz, B.P., Latimer, J.L., Mock, J.R., Vakevainen, M. \& Hansen, E. J. (2003). Haemophilus ducreyi requires an intact flp gene cluster for virulence in humans, Infect. Immun. 71, 7178-7182.

Sternberg, N. \& Hoess, R. (1983). The molecular genetics of bacteriophage P1, Annu. Rev. Genet. 17, 123-154.

Thomson, V.J., Bhattacharjee, M.K., Fine, D.H., Derbyshire, K.M. \& Figurski, D.H. (1999). Direct selection of IS903 transposon insertions by use of a broad host range vector: Isolation of catalase-deficient mutants of Actinobacillus actinomycetemcomitans, J. Bacteriol. 181, 7298-7307.

Tomich, M., Fine, D.H. \& Figurski, D.H. (2006). The TadV protein of Actinobacillus actinomycetemcomitans is a novel aspartic acid prepilin peptidase required for maturation of the Flp1 pilin and TadE and TadF pseudopilins, J. Bacteriol. 188, 68996914.

Tomich, M., Planet, P.J. \& Figurski, D.H. (2007). The tad locus: Postcards from the Widespread Colonization Island, Nat. Rev. Microbiol. 5, 363-375.

Tsai, C.C., McArthur, W.P., Baehni, P.C., Hammond, B.F. \& Taichman, N.S. (1979). Extraction and partial characterization of a leukotoxin from a plaque-derived Gram-negative microorganism, Infect. Immun. 25, 427-439.

Van Winkelhoff, A. J. \& Slots, J. (1999). Actinobacillus actinomycetemcomitans and Porphyromonas gingivalis in nonoral infections, Periodontol. 2000 20, 122-135.

Xu, Q., Christen, B., Chiu, H., Jaroszewski, L., Klock, H.E., Knuth, M.W., Miller, M.D., Elsliger, M., Deacon, A.M., Godzik, A., Lesley, S.A., Figurski, D.H., Shapiro, L. \& Wilson, I.A. (2012). Structure of pilus assembly protein TadZ from Eubacterium rectale: implications for polar localization, Mol. Microbiol. 83, 712-727.

Yue, G., Kaplan, J.B., Furgang, D., Mansfield, K.G. \& Fine, D.H. (2007). A second Aggregatibacter actinomycetemcomitans autotransporter adhesin exhibits specificity for buccal epithelial cells in humans and Old World primates, Infect. Immun. 75, 4440-4448.

Zambon, J.J. (1985). Actinobacillus actinomycetemcomitans in human periodontal disease, J. Clin. Periodont. 12, 1-20. 